\title{
ISCHAEMIC AND POSTISCHAEMIC PARAESTHESIAE IN POLYNEURITIS
}

\author{
BY \\ E. W. POOLE \\ From the Department of Neurology, Radcliffe Infirmary, Oxford
}

Paraesthesiae consequent on peripheral nerve compression or general limb ischaemia have attracted interest for over a century (Bastien and Vulpian, 1855), but their value in the study of nervous disease remains little explored. In a recent study (Poole, 1956) the paraesthesiae provoked in normal subjects by a standard ischaemic test were investigated in order to provide a background against which pathological conditions could be judged. This report concerns the responses elicited in subjects with polyneuritis.

Many features of paraesthesiae responses suggest their possible usefulness in the study of diffuse peripheral nerve disorders such as polyneuritis. The tingling, or " pins-and-needles ", which occurs during limb ischaemia, ischaemic paraesthesiae (I.P.), and which follow circulatory release, postischaemic paraesthesiae (P.I.P.), arises in sensory mechanisms subserving distal areas of the limb, and the larger fibres are probably mainly concerned. Such a preferential involvement is seen clinically, in polyneuritis from many causes, and experimentally, as in vitamin deficiency (Swank, 1940; Horwitt, Liebert, Kreisler, and Wittman, 1946), and intoxications, e.g., with triorthocresyl phosphate (Cavanagh, 1954). There is also evidence suggesting that such tests of iterative responsiveness may provide a sensitive index of nerve function. Sensation returns rapidly to normal after a period of limb ischaemia (Lewis, Pickering, and Rothschild, 1931), but paraesthesiae may not be elicited again to their full extent for many hours (Merrington and Nathan, 1949). Further, paraesthesiae may be lacking in certain areas in normal subjects without manifest sensory impairment, or be entirely absent as in the elderly (Poole, 1956). Thus it might be supposed that paraesthesiae responses should be readily disturbed in polyneuritis thereby providing a sensitive index of involvement and recovery. This theory was tested in two ways. Repeated examinations were made in conditions where some degree of recovery might be expected so that the significance and sensitivity of any paraesthesiae changes would be established by their subsequent regression along with general sensory recovery. Secondly, the responses in these and in other cases (either static or not available for follow-up) were compared with those elicited in comparable normal subjects (Poole, 1956). Since recovery was usually very slow this latter approach was more generally applicable. Fortunately the responses in normal subjects and in those with polyneuritis proved sufficiently uniform and divergent to render this method satisfactory, thus facilitating the simple clinical application of such tests.

\section{Material and Methods}

The cases studied are presented in three groups.

Group I.-This consisted of eight cases of acute toxic polyneuritis of unknown aetiology.

Group II.-This group contained 11 cases, the majority with polyneuritis of more chronic type, either of unknown aetiology or in association with other diseases. Included also are brief notes of the responses in less certain cases of polyneuritis in diabetes and porphyria, and in one case of Charcot-Marie-Tooth disease.

Group III.-This was five cases of megaloblastic anaemia, only one with a manifest polyneuritis. Neuropathies of various aetiologies have been included but a comprehensive survey of the effects of any single agent is not attempted.

Examinations were performed under warm, quiet circumstances wherever possible, and every effort made to avoid fatigue, the sensory testing being broken up with many intervals for rest and the ischaemic tests carried out when the subject was fresh or at an interval after sensory testing. The development and present status of the sensory and motor symptoms were recorded with special reference to the presence of spontaneous or "contact" paraesthesiae-like sensations. The usual clinical assessments of motor, reflex, and sensory functions were augmented by the use of graded nylon hairs to assess tactile perception (stimulus, $t,=$ bending thrust (g.) hair radius (mm.), weighted needles to provide standard pricking stimuli (Marshall, 1953), and tuning forks activated to standard degrees to assess vibration 
perception (Poole, 1954). Attempts were made to determine both the density and extent of sensory impairment so that serial examinations might give some objective measure of progress. Defects were judged against responses in more normal parts of the limb, performances in follow-up examinations, and experiences in normal subjects tested similarly.

Ischaemic tests in the upper limb were performed and assessed as previously described (Poole, 1956). Care was taken to ensure that the limb was warm and the interdigital cleft temperature was recorded. Special attention was paid to changes in any spontaneous or contact paraesthesiae initially present. In some cases observations were also made on the degree of postischaemic muscle twitching in the first dorsal interosseous muscle, a motor response analogous to P.I.P. but less readily induced (Kugelberg, 1944, 1948). An interval of at least 24 hours separated repeated tests on the same limb. In some instances lower limb occlusions were achieved without excessive discomfort, and the results have been noted. The technique was essentially similar to that for the arm, but the subject was lying flat and a cuff of $30 \mathrm{~cm}$. $\times 17 \mathrm{~cm}$. at 200 to $250 \mathrm{~mm}$. $\mathrm{Hg}$ pressure was employed high up on the thigh. The lower limb initially seemed, because of its greater susceptibility to disease processes, a more fruitful field for study, but the tests caused too much discomfort, appeared relatively less productive of P.I.P. than in the upper limb, and probably carried a greater risk of vascular damage. In all, over 120 tests were performed.

The duration of provocative ischaemia was important in determining the ensuing P.I.P. and muscle twitching. The presence of heightened muscle tenderness, the possible harmful effects of prolonged ischaemia, and the desire to provide a delicate assessment all favoured the minimal occlusion normally entirely adequate. A 10-minute upper arm occlusion was found to fulfil these requirements for P.I.P. in all except a minority of the elderly (Poole, 1956) and was then generally adopted. However, in the cases first studied occlusions were fixed individually as the greatest time between 10 and 20 minutes tolerated in the initial test. Later examinations of comparable duration might thus be readily achieved as muscle tenderness subsided with general recovery. However, the lack of P.I.P. norms for these varying occlusions created unnecessary difficulties in interpretation, though these could usually be overcome by reference to norms for the duration of P.I.P. in 10-minute tests (Poole, 1956). Prolonged occlusions had advantages in assessing postischaemic muscle twitching.

The timing of the initial and follow-up tests varied depending on the clinical state, progress, and availability for examination, some cases being followed for over two years. In acute disorders the initial test was performed only when the process had been clearly improving or stationary for at least one week to minimize the risk of aggravating the affection. This unfortunately left the earliest changes undefined, but it seemed wisest to postpone this until experience in the later phases established the precise features of interest. In fact, no adverse effects of occlusion tests were encountered and the responses in a limb serially examined over many mon were similar to those in the comparably affected opposte limb occluded finally for the first time.

\section{Results}

Group I (Acute Toxic Polyneuritis).-In all cas I.P. were absent or not clearly present, and P.IP. less marked than in normal subjects, even in some instances despite apparently complete subjective and objective sensory recovery. The occurren of spontaneous paraesthesiae-like impressions in the course of the disease did not favour the preduction of I.P. or P.I.P. Limited observations $\overline{\bar{\alpha}}$ post-ischaemic muscle twitching suggested that suळh responses were also depressed. Brief notes of the cases (in approximate order of age) follow. Nornal responses against which these results were judgẹd have been detailed previously (Poole, 1956), but $\exists$ t may be noted that clear I.P. were elicited in 1 young $(<31$ years) and the majority $(72 \%)$ af older normal subjects (31-60 years); while distift P.I.P. exceeding two minutes in duration were provoked by 10 -minute occlusions in all except minority of the oldest subjects ( $>60$ years).

Case 1.-This 16-year-old schoolgirl (R.I. 1790) became ill with a heavy cold and 10 days later (Augus $z$, 1953) developed a severe generalized paralysis requiting the use of intermittent positive pressure respiratio some extent for four weeks. Moderate distal sentsogy impairment was established when recovery permitted at the end of the first month. She made a comblere clinical recovery over the next seven months $\underset{7}{0}$ and ischaemic tests on the right arm gave the followii results (left essentially similar):-

\begin{tabular}{l|r|r|r|r|r|r}
\hline Weeks after onset & 8 & 9 & 11 & 15 & 28 & 1050 \\
Minutes of occlusion & 10 & 15 & 10 & 10 & 13 & $8 \cdot 5$ \\
I.P. (intensity) & 0 & 0 & 0 & 0 & 0 & $+\frac{7}{7}$ \\
P.I.P. (intensity) & 0 & 0 & 0 & \pm & + & $+\frac{1}{20}$ \\
Duration of P.I.P. (min.) & 0 & 0 & 0 & $<\frac{1}{2}$ & $1 \cdot 7$ & $\frac{2}{2}$ \\
\hline
\end{tabular}

Muscle power had completely recovered (in the upper limbs) by the 28th week (possibly by the 15 th weel The deep reflexes could be just elicited at the 28th week, but even at the 105th the triceps jerks remained depresseff. Sensory recovery was incomplete at the eighth week when doubtful spontaneous or contact paraesthesiae were still present from time to time and sensory testing su gested a minimal defect in 2-point discrimination; bout at later tests there was no subjective or objective i pairment.

As is shown above, I.P. were not elicited till the fingl examination when they appeared approximately normalin. Postischaemic paraesthesiae were similarly impaired, being at first entirely absent, and later dull and transie despite peripheral stimulation by tapping and pressure. However, in the final test (unfortunately limited $\overline{\bar{K}}$ eight and a half minutes' occlusion by discomfort) distinct, sharp P.I.P. were perceived-a response whidh might be taken as normal, though there were some minow 
atypical features in that the impressions were more marked in the palm than in the fingers, and peripheral stimuli (tapping and pressure) were required to produce sharp impressions.

Paraesthesiae responses were clearly severely impaired by the polyneuritis. Their recovery lagged behind that of ordinary sensation and was associated with the return of deep reflex responses.

Case 2.-This aircraftman, aged 21, developed respiratory infection on May 22, 1954, followed within a week by facial, palatal, and general muscle weakness, with paraesthesiae and sensory impairment in the limbs. Improvement was clearly evident one and a half weeks after the onset of nervous symptoms, and recovery was nearly complete after 10 weeks. Ischaemic tests during recovery gave the following results:-

\begin{tabular}{l|c|c|c|c}
\hline Weeks after onset & 6 & 8 & \multicolumn{2}{|c}{11} \\
& & & & $/ 1$ \\
Minutes of occlusion & $13(\mathrm{~L})$ & $13 \cdot 5(\mathrm{~L})$ & $14(\mathrm{~L})$ & $10(\mathrm{R})$ \\
I.P. (intensity) & 0 & 0 & + & + \\
P.I.P. (intensity) & 0 & \pm & + & + \\
Duration of P.I.P. (min.) & 0 & $<\frac{1}{2}$ & $4 \cdot 5$ & $2 \cdot 3$ \\
\hline
\end{tabular}

At the sixth week moderate general limb weakness persisted, with muscle tenderness and areflexia. The hands felt stiff and heavy, and sensation was abnormal, with a general "woolly" feeling and tingling on contact to a level 2 to 3 in. above the wrist. Testing showed slightly impaired tactile perception (3t) on the digits, but no change in pricking stimuli. Perception of passive movement at the distal interphalangeal joints was slightly defective as was vibration sense also. Two-point discrimination on the finger pulps showed occasional errors at $\frac{1}{2} \mathrm{~cm}$. separation. A 13-minute occlusion produced no paraesthesiae. His usual contact paraesthesiae were dulled relative to the other limb after four minutes and entirely eliminated by 12 minutes, only returning completely some 15 minutes after circulatory restoration (possibly partly by 3 minutes).

At the eighth week the upper limb power was almost normal, though the areflexia persisted. Sensation was not quite normal in that paraesthesiae on contact still persisted to some degree. Sensory examination showed minimal defects on the distal phalanges only. A 13.5 minute occlusion again gave no I.P. or P.I.P. However, there were some vague tingling impressions in the later stages of occlusion, and transient " beating" feelings in the fingers on circulatory release unaffected by peripheral stimulation.

At the 11th week upper limb power was essentially normal, and some deep reflexes were sluggishly elicited. Sensation was considered normal, and examination showed none of the previous minimal changes. Occlusions of 14 minutes (on the side usually tested) and 10 minutes (on the opposite) both gave slight I.P.; and P.I.P. also followed in the fingers, more severe and long-lasting after the longer occlusion. These P.I.P. were atypical in being only faintly spontaneously apparent and requiring peripheral stimulation to evoke sharp impressions. No muscle twitching was seen.

This case showed a progressive, though incom- plete, restoration of normal paraesthesiae responses (at this age), defects persisting despite apparently complete sensory recovery. Paraesthesiae-like impressions occurring as a result of the disease did not predispose to induced paraesthesiae with ischaemia and were readily depressed by circulatory occlusion. The lack of postischaemic muscle twitching was also suggestive of a defect in iterative responses, since occlusions of 15 minutes (Kugelberg, 1944) and even 10 minutes (Poole, 1956) provoked twitching in the majority of young normal subjects.

Case 3.-This schoolboy, aged 14 (R.I. 198793) had severe limb, bulbar, and facial paresis, but less severe sensory impairment. Muscle tenderness prevented ischaemic tests until late in the recovery, which was rapid once initiated. Two 10-minute occlusions were performed eight and 11 weeks after the onset, when spontaneous paraesthesiae had ceased and the upper limbs showed no subjective or objective sensory abnormality except a minimal defect in vibration perception at the terminal phalanges. Slight muscle paresis persisted, and the deep reflexes were absent at the first test and elicited doubtfully at the second. Moderate residual sensory and motor defects were clearly evident in the lower limbs. In both ischaemic tests I.P. were absent, but moderate P.I.P. (duration three minutes) and muscle twitching were provoked. The P.I.P. were relatively short-lived for this age, and only in the later tests were aggravated by peripheral stimuli. Lack of I.P. was clearly a significant departure from normal responses, and the short P.I.P. were in keeping with this defect (Poole, 1956).

Case 4.-This 17-year-old schoolgirl (R.I. 182462) developed, in association with glandular fever, a severe polyneuritis requiring artificial respiration to some extent for two weeks. Paraesthesiae and sensory defects were well marked, more severe on the left side. She made a very rapid recovery within six weeks of the onset, and ischaemic tests gave the following results:-

\begin{tabular}{l|c|c|c|c|c}
\hline Weeks after onset & 2 & \multicolumn{2}{|c|}{4} & \multicolumn{2}{|c|}{6} \\
Minutes of occlusion & $10(\mathrm{R})$ & $12(\mathrm{R})$ & $12(\mathrm{~L})$ & $8(\mathrm{R})$ & $8(\mathrm{~L})$ \\
I.P. (intensity) & $?$ & 0 & 0 & 0 & 0 \\
P.I.P. (intensity) & + & ++ & + & ++ & + \\
Duration of P.I.P. (min.) & $?<$ & $2 \cdot 2$ & $1 \cdot 8$ & $2 \cdot 2$ & $2 \cdot 5$ \\
\hline
\end{tabular}

The first test was performed in difficult circumstances during artificial respiration when there was probably some degree of alkalosis and nitrogen retention. Spontaneous paraesthesiae were present, and it was difficult to be certain if I.P. were induced, but P.I.P. followed to some extent. At the fourth week, despite rapid improvement with doubtful return of reflexes, considerable weakness persisted. Spontaneous paraesthesiae had largely gone, but the hands felt unnatural, especially the left, and there was slight impairment of all forms of sensation extending up to the wrist, more marked on the left. Occlusion tests (12 minute) produced no I.P. but short-lived P.I.P. followed in the fingers bilaterally, very feeble and curiously delayed in onset on the left. 
At the sixth week only slight paresis persisted distally and the deep reflexes could be clearly elicited. Sensation was not quite normal in the fingers, especially on the left side; a minimal defect was demonstrable in light touch on the left finger tips, and in the appreciation of passive movement at the terminal interphalangeal joints. Bilateral occlusions (unfortunately limited to eight minutes) provoked no I.P.; but moderate P.I.P. followed, less marked and delayed in onset on the left.

It was difficult to compare the initial test with later ones because of biochemical disturbances. In later tests the lack of I.P. was a consistent departure from normal, and P.I.P. were also reduced, at least in the earlier test. There was less impairment of P.I.P. than in many other cases, and it was noteworthy that the clinical recovery was rapid and the deep reflexes soon returned.

Case 5.-This labourer, aged 39 (R.I. 193125), developed severe motor and sensory defects in the limbs, the former recovering slowly almost completely over the next 15 months, but the latter tending to persist. Ischaemic tests performed during recovery gave the following results:-

\begin{tabular}{|c|c|c|c|c|c|c|}
\hline $\begin{array}{l}\text { Weeks after onset } \\
\text { Minutes of occlu- } \\
\text { sion } \\
\text { I.P. } \\
\text { P.I.P. } \\
\text { Duration of P.I.P. } \\
\text { (min.) }\end{array}$ & $\begin{array}{c}6 \\
15^{6}(\mathrm{~L}) \\
0 \\
? 0 \\
?<\frac{1}{2}\end{array}$ & $\begin{array}{c}9 \\
14^{(L)} \\
0 \\
? 0 \\
?<\frac{1}{2}\end{array}$ & $\begin{array}{c}14(\mathrm{~L}) \\
0 \\
0 \\
0\end{array}$ & $\begin{array}{c}30 \text { (R) } \\
\pm \\
0 \\
0\end{array}$ & $\begin{array}{c}10(\mathrm{~L}) \\
+ \\
+ \\
1-2\end{array}$ & $\begin{array}{c}0 \\
10(R) \\
\pm \\
\frac{+}{+} \\
2-3\end{array}$ \\
\hline
\end{tabular}

Spontaneous or contact paraesthesiae were present only at the first test, but moderate sensory defects persisted throughout. The motor power had returned almost to normal by the final test, but the deep reflexes remained absent. As is apparent, defects in I.P. and P.I.P. persisted throughout. Faint or doubtful I.P. were elicited only in the final tests. Some doubt arose as to whether there were any faint P.I.P. in the thermal postischaemic impressions in the early tests, but it was only in the final examinations that definite P.I.P. resulted. However, even these were abnormal in that there was little spontaneous activity, the impressions being mainly provoked by tapping and pressure on the fingers, and this response was very delayed in onset. Faint I.P. referred to areas with distinct residual sensory defects were not observed in other cases, and the prognostic significance of this is not yet apparent. Postischaemic muscle twitching was not seen after a 14-minute occlusion, suggesting similar defects in motor iterative responses.

Case 6.-This farmer, aged 42 (R.I. 145424), developed an acute polyneuritis two weeks after a sore throat with fever, but had been in brief contact with organic mercurial compounds six weeks previously. Motor involvement was severe but short of a degree requiring respiratory assistance; sensory impairment was relatively mild in the arms, more severe in the legs. Recovery after an initial relapse was moderately rapid in the arms, but slight motor and sensory defects were still evident in the legs 18 months later. Ischaemic tests during this period gave the follow ing consistently abnormal results:-

\begin{tabular}{|c|c|c|c|c|c|c|c|}
\hline $\begin{array}{l}\text { Weeks after onset } \\
\text { Minutes of occlusion } \\
\text { I.P. (intensity) } \\
\text { P.I.P. (intensity) } \\
\text { Duration of P.I.P. (min.) }\end{array}$ & $\begin{array}{l}5 \\
9 \cdot 5 \\
0 \\
0 \\
0\end{array}$ & $\begin{array}{l}7 \\
10 \\
? 0 \\
0 \\
0\end{array}$ & $\begin{array}{c}9 \\
11 \\
0 \\
\pm \stackrel{+}{<} 1\end{array}$ & $\begin{array}{l}11 \\
10 \\
0 \\
\pm \\
?<1\end{array}$ & $\begin{array}{l}18 \\
10 \\
0 \\
0 \\
0\end{array}$ & $\begin{array}{c}27 \\
10 \\
0 \\
0 \\
0\end{array}$ & 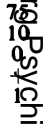 \\
\hline
\end{tabular}

Thus I.P. were not elicited except doubtfully in the second test in association with considerable discomfor Postischaemic paraesthesiae were not provoked wif any certainty till the final test, and even this short response was only faintly spontaneous and required peripher stimulation to provoke distinct pricking. These impaired responses were obtained despite the return of the uppor limb to subjective and objective sensory normality by the 27th week, if not before. An associated change wâs depression of the deep reflexes, but at the final examines tion biceps and supinator jerks were distinctly elicited.-

Cases 7 and 8. -Two elderly women (aged 52 and $6 \overrightarrow{2}$ who had had severe motor but slight distal senson. involvement (in the upper limbs) were examined whem there was little, if any, subjective or objective impairment, though spontaneous or contact paraesthesiae were presente occasionally in the finger tips. The deep reflexes were still not elicitable. Bilateral occlusion tests, limited muscle tenderness to eight minutes, gave no I.P., P.P., or muscle twitching. These results were not necessiriß a departure from normal at this age, though in keeping with usual polyneuritis responses. They show (as $\frac{1}{25}$ apparent in most cases) the importance of hatiog standard tests of minimal duration for applicatiog condjtions with heightened muscle tenderness.

Group II (Chronic, Recurrent, and Other Newire pathies).--In the majority of cases defects in I.PO P.I.P., and muscle twitching of the type found in Group I were observed. Thus only brief notes of the cases are presented except where possib significant additional points arose.

Case 9.-Recurrent acute polyneuritis in a mann aged 21 (R.I. 212192) without any precipitating cause. A 10-minute ischaemic test five weeks after the recurrence in the presence of moderate sensory defects gave I.P., P.I.P., or muscle twitching. "Contact" para. esthesiae-like sensations which were constantly present were readily depressed by ischaemia and totally abolished within seven minutes of occlusion, returning, at least part, within one minute of circulatory release. At the stage of ischaemia where such sensations were lacking the feeling in the occluded limb was considered to the more natural than in the unoccluded, as has been observed in other cases.

Case 10.-Polyneuritis in a woman aged 32 ( $\mathrm{R}$. 189300). In November, 1953, this housewife developed, insidiously, symmetrical limb weakness with paresthesiae and numbness in the fingers, feet and calves. Improvement was evident three months later and the paresis and reflex responses recovered (incompletely) over the next 16 months. Because of dense distal sensoif 
defects this case was studied specially to determine if at any stage of recovery paraesthesiae could be provoked in fibres yet to (or about to) re-establish full functional peripheral connexions with the numb areas on analogy with amputee results where paraesthesiae can be provoked in the phantom by stump ischaemia (Weddell and Sinclair, 1947; Merrington and Nathan, 1949; Poole, 1954). This might be of prognostic value, but, as experience with other cases showed, the readiness with which paraesthesiae responses were disturbed made it much more likely that their restoration would lag behind sensory recovery.

Ischaemic tests (15) were performed in the upper and lower limbs from the fifth to the nineteenth month after the onset. Ischaemic paraesthesiae, P.I.P., and muscle twitching were all provoked and the responses increased during recovery. Of more interest was the limited distal reference of paraesthesiae and their modification by peripheral stimulation. The dense distal sensory defects extended to a fairly sharp level $1-2 \mathrm{~cm}$. from the finger tip with no definite defect in more proximal phalanges save a slightly impaired vibration perception in the early stages. Ischaemic paraesthesiae, even when prominent, were confined to the palm. Post-ischaemic paraesthesiae extended little further than the proximal phalanges in earlier tests (16-18 minute occlusions) but later spread more distally towards the numb areas (10-16 minute occlusions). Peripheral stimuli of pressure on the proximal phalanges markedly aggravated the P.I.P. but on the distal phalanges had no effect. In proximal parts of the intermediate phalanges pressure could provoke P.I.P. not spontaneously apparent and, in the later stages of recovery, this response could be elicited slightly more distally. However, at no stage could paraesthesiae be provoked in areas of manifest sensory impairment nor in immediately adjacent proximal zones. Unfortunately sensory recovery was too limited to establish any order of recovery of sensation and paraesthesiae in the terminal parts. Nevertheless, the pattern of paraesthesiae reference on the limb strongly suggested that the recovery of paraesthesiae responses lagged behind that of sensation as ordinarily assessed.

Case 11.-Polyneuritis in a french-polisher aged 51 (S. 6758) developing insidiously without apparent cause, though toxic occupational factors must be considered. Motor and sensory defects were manifest subjectively and objectively in the legs, but the arms were never subjectively affected and examination disclosed only reflex depression and slight weakness without sensory impairment. Ischaemic tests 10 over a two-year period presented a uniform picture. Ischaemic paraesthesiae were absent and P.I.P. elicited transiently and feebly with 15-minute occlusions, those of 13 minutes and 10 minutes being ineffective. No postischaemic muscle twitching was seen. Apart from clearing of any weakness the clinical status of the arms remained unchanged, the reflex depression persisting. Comparable observations were made in the lower limb where gradual improvement in paresis and sensory defects was evident. Ischaemic paraesthesiae were consistently absent but short-lived P.I.P. were elicited by a 14-minute occlusion in the late recovery stages whereas previously this had produced no. response. It was noteworthy that upper limb paraesthesiae responses remained consistently out of keeping with the normal even though there had been no apparent sensory affection of these parts.

Case 12.-Polyneuritis with steatorrhoea in a woman aged 53 (R.I. 7143): symmetrical distal weakness and moderately severe sensory impairment developed insidiously in this long-standing case of steatorrhoea under vitamin and dietary treatment. Six ischaemic tests over a two-month period during which the clinical state showed little change gave consistent results: I.P. were absent; P.I.P. were not provoked by 10-minute occlusions but those of 14 minutes gave faint, dull, thrusting sensations in the fingers for two to three minutes. Since distinct sensory impairment was evident to mid-forearm level, the presence of even these dull P.I.P. was possibly surprising in comparison with other cases. It was noteworthy that, from the subsequent deterioration of this case, the lesions were probably not confined to the peripheral nerves (vide Smith, 1955).

Cases 13 and 14.-Chronic polyneuritis not certainly due to an underlying carcinoma in two women aged 65 and 68 (R.I. 193735; R.I. 186606): the younger had little upper limb sensory impairment, the elder severe; both had spontaneous paraesthesiae at times. In neither did repeated ischaemic tests of 10 to 20 minutes' duration over several months produce I.P., P.I.P., or muscle twitching.

Case 15.-Motor neuropathy with carcinoma of the lung in a man aged 61 (S. 10239): over a few months this man developed severe symmetrical wasting and weakness, maximal distally, without evident fasciculation or clear sensory impairment (allowing for slight ageing change in vibration perception). Deep reflexes were still elicitable. A 10-minute occlusion produced no I.P., P.I.P., or muscle twitching. This result was in keeping with a minority of normal responses at this age, and also with the responses seen in polyneuritis. However, this was also seen in subjects with similar extreme wasting from motor neuron disease.

Cases 16 and 17 (Diabetic Neuropathy).-Two diabetics (aged 58 and 61) had sensory defects and depressed reflexes in the lower limbs but no sensory impairment (or subjective abnormality) in the upper limbs, though the reflexes were also depressed or absent. A 10-minute upper arm occlusion in the older gave no I.P. or P.I.P. In the younger, though both were induced by an 11minute occlusion, they were referred predominantly to the palm and wrist rather than to the fingers, as is usual (for P.I.P. at least). The P.I.P. of less than two minutes' duration were shorter-lived and more feeble than might have been expected normally. These limited results, though possibly not greatly out of keeping with a minority of normal responses, tended towards the defects in polyneuritis and suggested paraesthesiae tests might contribute to the study of diabetic neuropathies.

Case 18.-Porphyria with neuropathy in a woman aged 20 (R.I. 168851): this girl, who had had symptoms 
of porphyria for one to two years and slight distal limb weakness for some months, rapidly developed symmetrical distal paresis with foot and wrist drop. However, the paresis did not spread, the reflexes were said to be unaltered, and no sensory symptoms or defects were recorded. When seen for ischaemic tests one month later, the paresis had been improving for two weeks; only moderate distal weakness persisted (predominantly in the dorsiflexors), the deep reflexes were elicited, and sensation appeared normal. A 14-minute occlusion gave clear I.P. and P.I.P. The normality of this result contrasted with cases of polyneuritis, but the pattern of nervous involvement was also different.

Case 19.-Charcot-Marie-Tooth disease in a woman aged 27 (R.I. 194340): this schoolteacher had severe wasting of all muscles distal to the upper third of the forearm with depressed or absent deep reflexes, and moderate sensory impairment distal to the wrist. Bilateral 10-minute tests gave no P.I.P. or muscle twitching (though there was little muscle to observe). No I.P. were induced on the right, but on the left some tingling began in the forearm four minutes after the occlusion and was thought to spread distally to involve all the fingers. There was no obvious reason for this asymmetry, and indeed the power and sensation were possibly better preserved on the right. Though lack of paraesthesiae might be attributed to sensory defects, muscle wasting in motor neuron disease has been found associated with similar defects.

Group III (Megaloblastic Anaemia).-In two patients in whom detailed observations and followup were possible, paraesthesiae responses were initially defective but returned towards normal with $B_{12}$ therapy, thus resembling acute toxic polyneuritis. These results are presented first, and then brief notes of three other patients in whom only limited studies could be made.

Case 20.-Polyneuritis and subacute combined degeneration with pernicious anaemia (R.I. 185685): this 65-year-old housewife with severe anaemia had had numbness, paraesthesiae, weakness, and unsteadiness in the legs for many months, and numbness and paraesthesiae in the hands for a few weeks before admission. Examination disclosed a mild polyneuritis in the arms. There was slight distal weakness, increased muscle tenderness, and depressed, though not absent, deep reflexes. Textures felt odd to the touch and there were occasional paraesthesiae in the fingers; but no great sensory impairment was demonstrable, there being only slight defects in tactile (3t) and vibration perception at the terminal phalanges, and in the detection of passive movement at the terminal interphalangeal joints. However, two points could not be discriminated with complete accuracy at $1 \mathrm{~cm}$. separation on the pulps. The lower limbs showed considerable objective and subjective sensory and motor disturbances distally with clear evidence of cord lesions. Ischaemic tests were performed in both upper and lower limbs before therapy and over the ensuing seven months. The arm results were as follows:-

\begin{tabular}{|c|c|c|c|c|c|c|c|c|c|}
\hline Weeks, relative to & $-\frac{1}{2}$ & $+\frac{1}{2}$ & +1 & +2 & +3 & $+5 \frac{1}{2}$ & $+7 \frac{1}{2}$ & +12 & 28 \\
\hline $\begin{array}{l}\text { Minutes of occlu- } \\
\text { sion }\end{array}$ & 12 & 12 & 14 & 17 & 21 & 20 & 20 & 20 & \\
\hline $\begin{array}{l}\text { I.P. (intensity) } \\
\text { P.I.P. (intensity) } \\
\text { Duration of P.I.P. } \\
\quad \text { (min.) }\end{array}$ & $\begin{array}{l}0 \\
0 \\
0\end{array}$ & $\begin{array}{l}0 \\
0 \\
0\end{array}$ & $\begin{array}{l}0 \\
0 \\
0\end{array}$ & $\begin{array}{l}\mathbf{0} \\
0 \\
0\end{array}$ & $\begin{array}{l}\mathbf{0} \\
\mathbf{0} \\
\mathbf{0}\end{array}$ & $\begin{array}{c}0 \\
+ \\
3-4\end{array}$ & $\begin{array}{c} \pm \\
+ \\
4\end{array}$ & $\begin{array}{c}+ \\
++\end{array}$ & 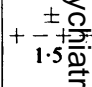 \\
\hline Haemoglobin (\%) & 37 & 46 & 50 & 86 & - & 85 & 89 & - & \\
\hline
\end{tabular}

As is seen, I.P. and P.I.P. were not elicited before therapy nor in the succeeding month, even though there was considerable subjective improvement in that spoiftaneous paraesthesiae disappeared and only the finger tips felt slightly odd. Moreover, objectively, the power increased, reflexes became more definite, and sensogy testing showed none of the previous defects except a minimal one in vibration perception. During the secoßd month distinct, though mild, P.I.P. were elicited for the first time, referred mainly to the palm and proxingal phalanges. The upper limbs were considered subjectivety normal except for a very doubtful finger tip change sensation without objective defects. During the third month when the hands were entirely natural slight ISP. were also induced (though only in the one test referred to the fingers), and increased P.I.P. were elicited referrod to all phalanges. With the final standard 10-minute test I.P. were not clearly present but P.I.P. were induced throughout the hand and were aggravated by preste and movement.

Parallel studies in the leg showed, in the first month,

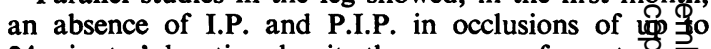
24 minutes' duration despite the presence of spontapeois paraesthesiae-like sensations. However, from the siond month onwards, P.I.P. consistently followed 20-minu occlusions and after the third month I.P. were as apparent even though distinct sensory defects persisted along with a spastic paresis (and absent ankle jerks)

The recovery of normal paraesthesiae responses

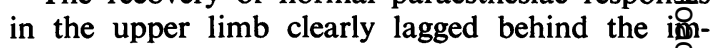
provement in the blood count and followed môre closely the symptoms of subjective sensory no․mality. The initial lack of distal reference possibly significant in the pattern of recovery. The appearance of I.P. later than P.I.P. was in keepi莫g with the long duration of provocative ischaenia and the usual findings in polyneuritis. The abse $\overline{x c e}$ of clear I.P. in the final test may be a reflection. of the feebleness of the sensations normally at this and the less satisfactory examination conditions in the final follow-up. In the lower limb, despgte persistent residual defects, there was a distikict recovery presumably due to the regression of polyneuritis, but it was not possible to ascertain if the residual sensory defects were entirely detarmined by cord lesions.

Case 21.-Post-gastrectomy megaloblastic anaemiăin a man aged 55 (R.I. 188738): a partial gastrectomy \$ुor 
gastric ulcer was performed in 1941 and 12 years later investigation disclosed a megaloblastic anaemia with deficient $B_{12}$ absorption. There were no nervous symptoms, and motor, reflex and sensory examinations were entirely normal apart from doubtfully increased muscle tenderness and a minimal defect in vibration perception in the legs and possibly in the arms. Ischaemic tests on the left arm before and after $B_{12}$ therapy gave the following results:-

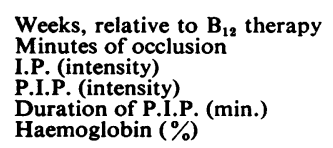

\begin{tabular}{c|c}
$-\frac{1}{2}$ & \\
14 & 0 \\
0 & \\
0 & \\
48 &
\end{tabular}

\begin{tabular}{c|c}
+2 & + \\
19 & \\
\pm & \\
+ & + \\
2 &
\end{tabular}

\begin{tabular}{c|c}
+3 & +48 \\
$16 \frac{1}{2}$ & 10 \\
+ & ++ \\
++ & ++ \\
75 & $2 \cdot 7$ \\
75 & 105
\end{tabular}

Thus, whereas both I.P. and P.I.P. were lacking before therapy, these were elicited with increasing vigour following $B_{12}$ administration. Parallel studies in the lower limb showed very similar changes. Ischaemic paraesthesiae and P.I.P. were lacking initially but P.I.P. were feebly evoked after two weeks' therapy and to a greater extent after three weeks when I.P. were also reported. Thus, as in cases of polyneuritis, defects in paraesthesiae could occur in the absence of objective or subjective sensory changes and, with $\mathrm{B}_{12}$ therapy, recover in association with improvement in the blood count, without obvious change in the sensory status.

Case 22.-Mild pernicious anaemia in a woman aged 56 (R.I. 110330) without symptoms or signs of nervous lesions. A 10-minute occlusion before therapy (haemoglobin $68 \%$ ) gave doubtful I.P. and faint P.I.P. (duration less than 2 minutes) referred patchily to digits III and V.

Case 23.- Severe pernicious anaemia in a woman aged 68 (R.I. 77218) without symptoms or signs of nervous lesions: a 10-minute occlusion before therapy (haemoglobin $40 \%$ ) provoked no I.P. but minimal (or doubtful) P.I.P. followed, amounting to slight throbbing in the terminal pulps for two to three minutes, unaffected by peripheral stimuli.

Case 24.-Mild obscure megaloblastic anaemia in a woman aged 50 (R.I. 189298), not secondary to manifest steatorrhoea: there were no symptoms indicating nervous involvement but she was a dull witness. The deep reflexes were all sluggish and one plantar response equivocal, but there was no certain sensory impairment. Five occlusions of 16 to 20 minutes in the upper and lower limbs during one month's $B_{12}$ therapy (when the haemoglobin level rose from 70 to $80 \%$ ) provoked no paraesthesiae or muscle twitching.

These three cases showed responses either less than or of the minimal type found in normal. subjects. While it was clearly unwise to apply " normal" criteria in any strict fashion in the absence of complete follow-up, reduced iterative responses were in keeping with other cases of this series.

\section{Discussion}

This study confirms the view that paraesthesiae responses are severely disturbed in polyneuritis. Such depression of iterative responses provides a sensitive index of preceding affection, as is shown in many features such as the persistence of paraesthesiae defects in the absence of subjective or objective sensory impairment, the regression of such defects without obvious change in sensory status, and the failure of paraesthesiae to be elicited in normal areas proximal to affected parts (e.g., in the palm when only the finger tips are clinically abnormal). The influence of age on normal responses is of value in interpreting the results, and indeed the defects in polyneuritis are those to be expected from the type of change seen in ageing (Poole, 1956) and in repeated limb ischaemia (Weddell and Sinclair, 1947; Merrington and Nathan, 1949). Thus, as in ageing, I.P. are more frequently totally lacking than " 10-minute" P.I.P., but in such circumstances the latter are usually relatively short-lived. Since 10-minute P.I.P. are much more intense than I.P. this relationship suggests some common factor impairing the most responsive elements or producing a general decrease in iterative activity. It is noteworthy that the polyneuritis results are in keeping with the general working rule proposed from ageing studies that, if I.P. are clearly present, P.I.P. will follow a 10-minute occlusion, a rule which also appears to hold in cerebral and cord lesions (Poole, 1954).

Observations on muscle twitching, though limited, support the view that such iterative responses are also impaired, even though the only associated clinical defect might be depression of deep reflexes. Muscle twitching was never unequivocally provoked in the absence of P.I.P., thus preserving the normal relationships between sensory and motor responsiveness (Kugelberg, 1944 and 1948; Skoglund, 1942; Poole, 1956), but electromyographic studies would be needed for conclusive observations in this field.

In megaloblastic anaemia decreased paraesthesiae responses without manifest sensory impairment may be due to subclinical neuropathy from $\mathrm{B}_{12}$ deficiency, but the influence of anaemia per se has not been established in these preliminary studies. However, tests in 13 subjects with secondary anaemia (haemoglobin $55-74 \%$ ) showed little sign of impaired responses (Poole, 1956), and in the two megaloblastic cases studied most completely (20 and 21$)$ the return of normal paraesthesiae was related more to the severity of the initial nervous lesions than to the restoration of the blood count. Nevertheless, the frequent occurrence of paraesthesiae as symptoms of severe anaemia and the inhibiting effect of recurrent ischaemia on I.P. and P.I.P. both suggest that disturbed iterative responses might well be found in 
severe anaemia if occlusion tests can be safely applied.

From the clinical viewpoint the present results are of interest not only in defining the presence and progress of neuropathies in many diseases (intoxications, vitamin deficiencies, alcoholism, malignant disease, diabetes, leprosy, etc.) but also in the study of different types of peripheral nerve dysfunction. Whereas severe depression of paraesthesiae responses appears characteristic of diffuse polyneuritis, normal or exaggerated responses may occur in focal lesions, e.g., carpal tunnel syndrome (Gilliatt and Wilson, 1953), in general hyperexcitable states, e.g., tetany (Kugelberg, 1944), and possibly also in interstitial neuritis since prematurely-occurring I.P. have been described in sciatica (Marshall, 1952). It is noteworthy that gross depression of paraesthesiae responses in association with slight sensory defects (or spontaneous paraesthesiae-like sensations) has not been found to be the rule in preliminary studies of cord and cerebral lesions (Poole, 1954). The prognostic value of paraesthesiae assessments remains uncertain. Little has been found to favour the possibility that paraesthesiae might be provoked in proximal parts of recovering fibres and thereby indicate potential recovery (vide Case 10). With few limited (but perhaps significant) exceptions recovery of iterative responsiveness lagged behind full clinical sensory recovery in the area concerned.

It is not possible to discuss the many problems raised by this study but two may be briefly noted. One feature is that spontaneous paraesthesiae or similar sensations evoked by surface contact do not favour the production of I.P. or P.I.P., though they are obviously influenced by peripheral ischaemia. As has been pointed out, this forms a contrast with other conditions where spontaneous paraesthesiae are a feature. The sensory mechanisms involved in such spontaneous sensations may not be those participating in ischaemic iterative responses, but their susceptibility to ischaemia (and activation by pressure) makes this likely. Another problem is the precise basis for the defects in paraesthesiae. In polyneuritis (as in ageing) decreased responses may occur without manifest sensory impairment, just as reflex depression may persist despite " full " motor recovery. Indeed these associated paraesthesiae and reflex defects might suggest a common basis, that is, an afferent basis for reflex depression and a proprioceptive share in paraesthesiae responses. $\mathrm{Ob}-$ viously, defects in paraesthesiae occurring in the absence of clear sensory deficits may be due to the crudity of the latter and the delicacy of the former assessments in detecting a functional loss of fibrs. However, the question arises whether there isōa stage at which induced iterative fibre activity may be impaired though function via end-organ stimufation is relatively intact. Slight disturbances $\mathrm{dn}$ myelination might provide a basis for this, though many other changes have to be borne in mind. The decreased responses in polyneuritis represent a tre $\vec{n} d$ towards the motor type of reaction to ischaenia (Skoglund, 1942; Kugelberg, 1944 and 1948) and the factors determining the accommodation agd repetitiousness of various fibres are ill-defined. Electrophysiological studies in clinical and expesimental neuropathies may clarify this and circumve some of the difficulties inherent in clinical sensary investigations.

\section{Summary}

Ischaemic and postischaemic paraesthesiae have been studied in cases of polyneuritis and mega $\overline{0}-$ blastic anaemia. The responses elicited successivęy over many months have been contrasted with clinical recovery, and compared with those fouitd in normal subjects. These observations confien that such paraesthesiae are profoundly distuofbed in polyneuritis and thereby provide a sensitive of involvement and recovery. Postischaemic miscte twitching responses appear similarly depressed. OTe significance of these defects in iterative responsessis briefly discussed.

I wish to thank Dr. W. Ritchie Russell an Dr. C. W. M. Whitty for permission to study patients unfer their care, and for advice and criticism of the text. Some of the cases were kindly provided by Prof. L. J. Witts, and Drs. A. M. Cooke, E. M. Buzzard, J. Kidd, a. P. Mallam. Financial assistance from a Henry Goodgetr Scholarship is gratefully acknowledged.

\section{REFERENCES}

Bastien, J. B., and Vulpian, A. (1855). Gaz. méd., Paris, 3 ser, Cavanagh, J. B. (1954). Journal of Neurology, Neurosurgery Cavanagh, J. B. (1954).

Gilliatt, R. W., and Wilson, T. G. (1953). Lancet, 2, 595.

Horwitt, M. K., Liebert, E., Kreisler, O., and Wittman, P. (1960). Science, 104, 407.

Kugelberg, E. (1944). Acta. physiol. scand., 8, Suppl. 24.

Lewis, T. Pickering. Neurol. Psychiat. (Chicago), 60, 140.

Marshall, J. (1952). Journal of Neurology, Neurosurgery ejsd Psychiatry, 15, 242.

-, (1953). Clin. Sci., 12, 247.

Merrington, W. R., and Nathan, P. W. (1949). Journal of Neurology, Neurosurgery and Psychiatry, 12, 1.

Poole, E. W. (1954). Unpublished observations. (1956). Journal of Neurology, Neurosurgery and Psychiatry, $19,148$.

Skoglund, C. R. (1942). Acta. physiol. scand., 4, Suppl. 12. Smith, W. T. (1955). Excerpta. med. (Amst.), Sect. VIII, 8, 860. D Wwank, R. L. (1940). J. exp. Med., 71, 683. Neurosurgery and Psychiatry, 10, 26. 